\title{
Study of Methylene Blue Ototoxicity in the Guinea Pig
}

\author{
Sarah Belhassen ${ }^{\mathrm{a}}$, Musaed Alzahrani ${ }^{\mathrm{a}}$, Marc-Elie Nader ${ }^{\mathrm{a}}$, \\ Louis Gaboury ${ }^{\mathrm{b}}$, Issam Saliba ${ }^{\mathrm{a}, \mathrm{c}}$
}

\begin{abstract}
Background: Methylene blue is widely used in the medical field, especially as a blue dye for staining. It is also used as a photosensitizing agent in antimicrobial photodynamic therapy, which once photoactivated is effective for the eradication of several multi-resistant bacteria. The objective of this study was to investigate the ototoxic potential of methylene blue and precise its use in otology.
\end{abstract}

Methods: It was a prospective animal study performed on guinea pigs in our tertiary medical center. We divided the animals into two groups: an experimental group and a control group, who underwent a series of three intratympanic (IT) injections. In the control group $(\mathrm{n}=$ 10), they received injections of gentamicin in one ear (positive control) and normal saline in the contralateral ear (negative control). The experimental group $(n=10)$ received injections of methylene blue in one ear, compared to injections of normal saline in the contralateral ear. We conducted auditory-evoked brainstem response (ABR) before and 1 week after the injection series. Once this is completed, the cochlea was dissected and caspase- 3 was analyzed by immunohistochemistry.

Results: The mean difference of hearing loss in the methylene blue group compared to normal saline was $1.50 \mathrm{~dB}$, and it was not shown to be statistically significant $(\mathrm{P}=0.688)$. For the positive control group, which received IT injections of gentamicin, the mean threshold of hearing loss difference for all the frequencies combined was $66.25 \mathrm{~dB}(\mathrm{P}<0.001)$. Furthermore, uptake of caspase-3 by immunohistochemistry (apoptotic marker) was negative in our group, which received injections of methylene blue.

Conclusion: In light of our results, IT injections of methylene blue did not demonstrate an ototoxic potential. We recommend further studies to precise its use in the otologic field.

Keywords: Hearing loss; Intratympanic injection; Methylene blue; Ototoxicity; Immunohistochemistry

Manuscript submitted July 20, 2017, accepted August 29, 2017

aDivision of Otolaryngology-Head and Neck Surgery, University of Montreal, Sainte-Justine University Hospital Center (CHU SJ), Montreal, Quebec, Canada

${ }^{b}$ Department of Pathology, University of Montreal, Quebec, Canada ${ }^{\mathrm{c} C}$ Corresponding Author: Issam Saliba, Division of Otolaryngology-Head and Neck Surgery, 1560, Rue Sherbrooke East, Montreal, Qc, H2L 4M1, Canada. Email: issam.saliba@umontreal.ca

doi: https://doi.org/10.14740/jocmr3136w

\section{Introduction}

Methylene blue is an aromatic chemical compound mainly used in biology and chemistry. Heinrich Caro first synthesized it in 1876 and since, it has been widely used in the medical field [1]. Methylene blue is used for the treatment of methemoglobinemia [2], septic shock [3], and hepatopulmonary syndrome [4], or as an antimalarial agent [5]. It is also used as an antidote for the treatment of ifosfamide toxicity, or for heparin neutralization in patients presenting with protamine allergy.

In the otolaryngology field, methylene blue is used as a blue dye for staining. In fact, it has been shown to be a safe and effective method for localizing abnormal parathyroid gland preoperatively [6], or for sentinel lymph node localization. Furthermore, recent studies suggest that it can induce selective cancer cell apoptosis, especially when used together with photodynamic therapy for cancer [7].

Methylene blue also has antiseptic properties. Combined with light, methylene blue has been shown to be an effective antimicrobial agent (photodynamic antimicrobial therapy) [8]. With the emergence of resistant strains of bacteria, the treatment of many diseases, including chronic otitis media, is becoming increasingly difficult. This has stimulated the search for new alternative approaches, such as antimicrobial treatments.

The avenue of a new technological approach, antimicrobial photodynamic therapy (PDT), uses photo-activation of agents called photosensitizers, such as methylene blue. Once photoactivated, they acquire antimicrobial properties effective even against resistant germs such as methicillin-resistant Staphylococcus aureus (MRSA) [9]. This method is more widely used for oral pathologies [10], but its application in the otologic field remains to be defined. Many studies have demonstrated the efficacy of photodynamic antimicrobial chemotherapy using methylene blue as a photosensitizer [11-13] including effectiveness against most common pathogens found in chronic otitis, such as Staphylococcus aureus or Pseudomonas aeruginosa [9, 14-16].

Methylene blue, as a blue dye, discolors urine green and turns skin and mucosa bluish. Methylene blue toxicity remains a rare condition, but it has been associated with different adverse effects when used in high doses, such as cardiac, renal and pulmonary problems [7].

No previous study explored the ototoxic potential of methylene blue. Given the increasing use of methylene blue in the medical field, we wanted to establish its medical safety as well as its use in the otologic field. 


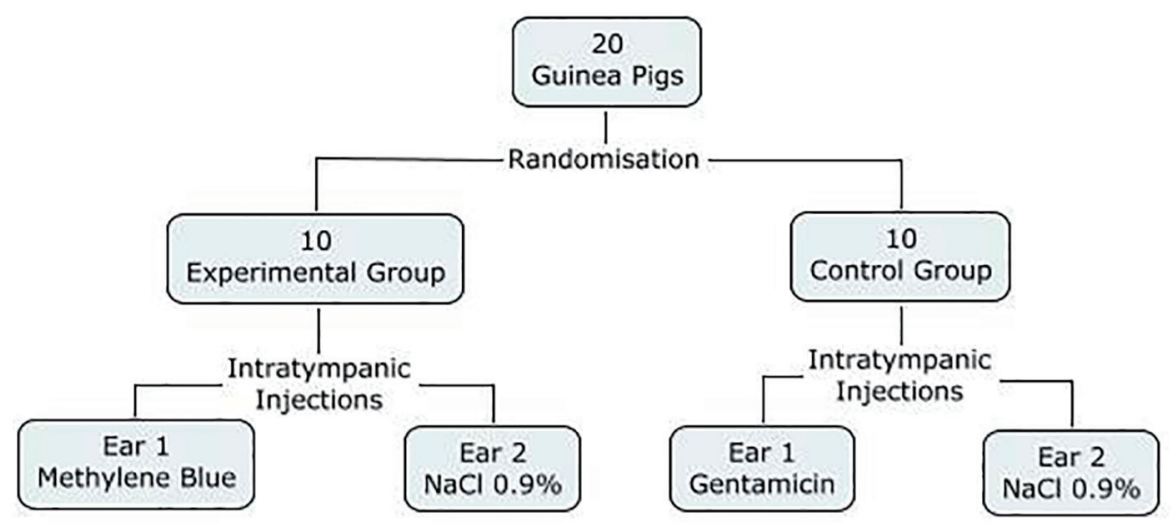

Figure 1. Study design.

The main objective of this study was to determine the ototoxic potential of methylene blue, and to evaluate its safety in otology.

\section{Materials and Methods}

\section{Animal model}

Twenty guinea pigs were used for this study, with weight varying between 250 and $300 \mathrm{~g}$. We chose this animal model as they have a relatively large middle ear and tympanic eardrum, which facilitate manipulations, and because their functional anatomy resembles that of humans. Furthermore, our laboratory has a wide experience in handling of these animals.

This study was approved by our institutional review board. All manipulations on animals were made in accordance with the guidelines of the Canadian Council on Animal Care and standards of institutional Animal Care Committee.

\section{General procedure and groups assignments}

All the guinea pigs were randomly assigned to two groups, an experimental group and a control group. The experimental group received intratympanic (IT) injections of methylene blue $(300 \mu \mathrm{g} / \mathrm{mL})$ in one ear, and normal saline $(\mathrm{NaCl} 0.9 \%)$ in the contralateral ear. Varying concentrations of methylene blue have been described for the use of photodynamic therapy, and we chose this concentration as it was one of the highest described in the literature. In the control group, injections of gentamicin $(40 \mathrm{mg} / \mathrm{mL})$ were achieved in one ear (positive control), compared to injections of $\mathrm{NaCl} 0.9 \%$ in the contralateral ear (negative control) (Fig. 1).

A total of three sets of IT injections were performed over 10 days: the first one at day 0 , the second at day 5 and the third at day 10. Each IT injection was done under a surgical microscope using a Withacre Spinal needle 27G, in the posteroinferior quadrant of the tympanic eardrum.

All animals survived until the end of the study, and no ear infection was noted.

\section{Anesthetic protocols}

Induction of anesthesia and maintenance both for the auditory brainstem responses (ABRs) recordings and the IT injections were achieved with 2-3\% isoflurane. Before euthanizing the animals, a deep anesthesia was achieved with 5\% isoflurane.

\section{ABR recordings}

ABR recording for each animal's ear was achieved 1 week before the first IT injection, and it was repeated 1 week following the last IT injection. External auditory canals were inspected before the recordings and cleaned of any cerumen debris if present. They were all recorded using the same Nicolet Bravo device (Nicolet Bravo System, Nicolet Biomedical, Madison, WI, USA). A total of four subcutaneous needle electrodes were used to record the electrical activity: one inverting electrode was inserted on the vertex, two non-inverting electrodes were placed over the left and right mastoid processes, and one ground electrode was inserted over the back of the animal. Before each $\mathrm{ABR}$ recording, the impedance was verified and accepted if inferior to $3 \mathrm{k} \Omega \mathrm{s}$. Tone bursts stimuli at 2,000, 4,000, 6,000 and $8,000 \mathrm{~Hz}$ were generated. The electrophysiological threshold was defined as the lowest intensity showing a visible $\mathrm{V}$ wave at least three times.

\section{Euthanasia protocol and cochlea's dissections}

After achieving a deep general anesthesia using 5\% isoflurane, all animals were euthanized. The middle ear was then exposed by removing the tympanic membrane and the cartilaginous portion of the external auditory canal. Each cochlea's was then dissected for a total of 40 specimens.

\section{Tissue preparation}

After dissections, samples were placed in $10 \%$ buffered formalin for at least $48 \mathrm{~h}$ for fixation. Once fixed, specimens were 
Table 1. Auditory Brainstem Responses Thresholds in Decibel for the Control Group (Normal Saline vs. Gentamicin) Before and After the Completion of the Intratympanic Injections

\begin{tabular}{|c|c|c|c|c|c|c|}
\hline \multirow{2}{*}{ Frequencies } & \multicolumn{3}{|c|}{ Normal saline } & \multicolumn{3}{|c|}{ Gentamicin } \\
\hline & Before & After & Hearing loss & Before & After & Hearing loss \\
\hline $2,000 \mathrm{~Hz}$ & $29.00 \pm 6.58$ & $37.00 \pm 8.88$ & $8.00 \pm 8.23$ & $30.50 \pm 6.85$ & $105.00 \pm 28.28$ & $74.50 \pm 28.62$ \\
\hline $6,000 \mathrm{~Hz}$ & $24.50 \pm 11.17$ & $25.50 \pm 7.98$ & $1.00 \pm 5.68$ & $19.00 \pm 10.49$ & $86.00 \pm 23.19$ & $67.00 \pm 26.06$ \\
\hline $8,000 \mathrm{~Hz}$ & $9.50 \pm 10.39$ & $7.50 \pm 8.90$ & $-2.00 \pm 5.37$ & $4.00 \pm 5.68$ & $66.50 \pm 25.83$ & $62.50 \pm 23.07$ \\
\hline
\end{tabular}

Thresholds are shown in decibels \pm standard deviation.

decalcified using TBD2 solution for 48 - $72 \mathrm{~h}$. Processing of the samples was initiated with dehydration using three alcohol baths with growing concentrations $(70 \%, 85 \%$, and $90 \%)$ to prevent tissue damage, and then water was completely removed by three final absolute alcohol baths. Three toluene baths then replaced the alcohol present, and all specimens were finally immerged in hot wax baths at $44-60^{\circ} \mathrm{C}$ to solidify the tissue. The specimens were embedded in molds filled with hot paraffin. Once the mold was fixed, tissue section at $4 \mu \mathrm{m}$ was achieved using a microtome.

\section{Histology and immunohistochemistry (IHC) analysis}

Routine histology staining was initially done using hematoxylin-eosin coloration to assess the global architectural structures. To identify cell apoptosis, we performed IHC analysis using the detection of the caspase- 3 antibody. The caspase-3 protein, which is encoded by the CASP3 gene, is a member of the Caspase family (cysteine-aspartic acid protease). These proteins play a central role in the execution-phase of cell apoptosis.

Apoptosis is a process, in which cells activate an intrinsic suicide mechanism that destroys themselves. The proteases that mediate this execution are called capases (cysteinyl-aspartic acid proteases). Apoptosis has gained central importance in the study of many biological processes, including neoplasia, neurodegenerative diseases, and development. We used the cleaved form of caspase- 3 which detects endogenous levels of the large fragment $(17 / 19 \mathrm{kDa})$ of activated caspase- 3 resulting from cleavage adjacent to Asp175. Activation of caspase-3 requires proteolytic processing of its inactive zymogen into activated p17 and p12 subunits. Cleavage of caspase- 3 requires aspartic acid at the $\mathrm{P} 1$ position. This antibody does not crossreact with other cleaved caspases.

Antigen detection, in tissues and cells, is a multi-step immunohistochemical process. The initial step was a heatinduced-epitope-retrieval incubation to improve the detection of our antibody staining on formalin fixed paraffin embedded tissue sections, using Standard Cell Conditioning 1 (Ventana Medical Systems \# 950-124, Roche) [17], then a following step bound the primary antibody to its specific epitope. Here the primary antibody was a rabbit polyclonal anti-cleaved caspase-3 from Biocare Medical used at 1/50 for $60 \mathrm{~min}$ at room tempera- ture that was labeled with the antigen, caspase-3. The section was not blocked before incubation with the first antibody. After labeling the antigen with a primary antibody, a secondary antibody was added to bind to the primary antibody. In this study, fluorescein isothiocyanate (FITC) labeled secondary antibody (Fluorescein (FITC)-AffiniPure Donkey Anti-Rabbit, 1/100) was added to bind to the primary antibody for 30 min at room temperature. Finally ProLong ${ }^{\text {TM }}$ Gold Antifade Mountant with DAPI (4',6-diamidino-2-phenylindole) was added to the section and slide was cover slipped. The whole immunofluorescence staining was achieved on the Discovery XT platform (Ventana Medical Systems, Roche) at the Institute for Research in Immunology and Cancer of our university institution.

\section{Statistical analysis}

The number of animals needed to find a clinical significance $(10 \mathrm{~dB})$ was initially determined using the method of Snedecor (1989) [18]. Statistical analysis was performed using a threeway ANOVA variance analysis. The effects of the frequency $(2,000,4,000,6,000$ and 8,000 Hz), the side treated (right vs. left) and the product injected (methylene blue, gentamicin, and normal saline) were measured. No interactions between these variables were found, and each of them was thus studied separately. A P-value inferior to 0.05 was considered significant.

\section{Results}

\section{Auditory brainstem responses}

\section{Descriptive analysis: ABR in control groups}

For the negative control group, which received IT injections of normal saline, the mean threshold before the IT injections was $23.50 \mathrm{~dB}$. After the completion of the IT injections, the mean threshold remained at $25.88 \mathrm{~dB}$.

For the positive control group, which received IT injections of gentamicin, the mean initial threshold was $20.25 \mathrm{~dB}$. At the end of the sets of the IT injections, the threshold was at $88.88 \mathrm{~dB}$. All the ABR recordings of the control groups regard- 
Table 2. Auditory Brainstem Response Thresholds in Decibel for the Methylene Blue Group Before and After the Intratympanic Injections

\begin{tabular}{|c|c|c|c|c|c|c|}
\hline \multirow{2}{*}{ Frequencies } & \multicolumn{3}{|c|}{ Normal saline } & \multicolumn{3}{|c|}{ Methylene blue } \\
\hline & Before & After & Hearing loss & Before & After & Hearing loss \\
\hline $2,000 \mathrm{~Hz}$ & $32.50 \pm 8.58$ & $33.00 \pm 11.60$ & $0.50 \pm 6.85$ & $38.00 \pm 6.32$ & $41.00 \pm 13.08$ & $3.00 \pm 14.18$ \\
\hline $6,000 \mathrm{~Hz}$ & $14.50 \pm 4.97$ & $16.50 \pm 13.34$ & $2.00 \pm 12.74$ & $17.50 \pm 4.86$ & $20.00 \pm 11.79$ & $2.50 \pm 12.08$ \\
\hline $8,000 \mathrm{~Hz}$ & $-0.50 \pm 5.50$ & $0.00 \pm 10.54$ & $0.50 \pm 10.66$ & $2.50 \pm 6.35$ & $5.00 \pm 11.55$ & $2.50 \pm 11.37$ \\
\hline
\end{tabular}

Thresholds are shown in decibels \pm standard deviation.

ing each frequency are shown in Table 1.

\section{Descriptive analysis: ABR in experimental group}

In the experimental group, the ears treated with normal saline presented a mean initial ABR recording of $17.63 \mathrm{~dB}$. After the last injections, the threshold was at 18.88 . For the ears treated with methylene blue, the mean initial ABR recording was $21.38 \mathrm{~dB}$, and stayed at $24.13 \mathrm{~dB}$ by the completion of the IT injections. All the ABR recordings of the experimental group regarding each frequency are shown in Table 2.
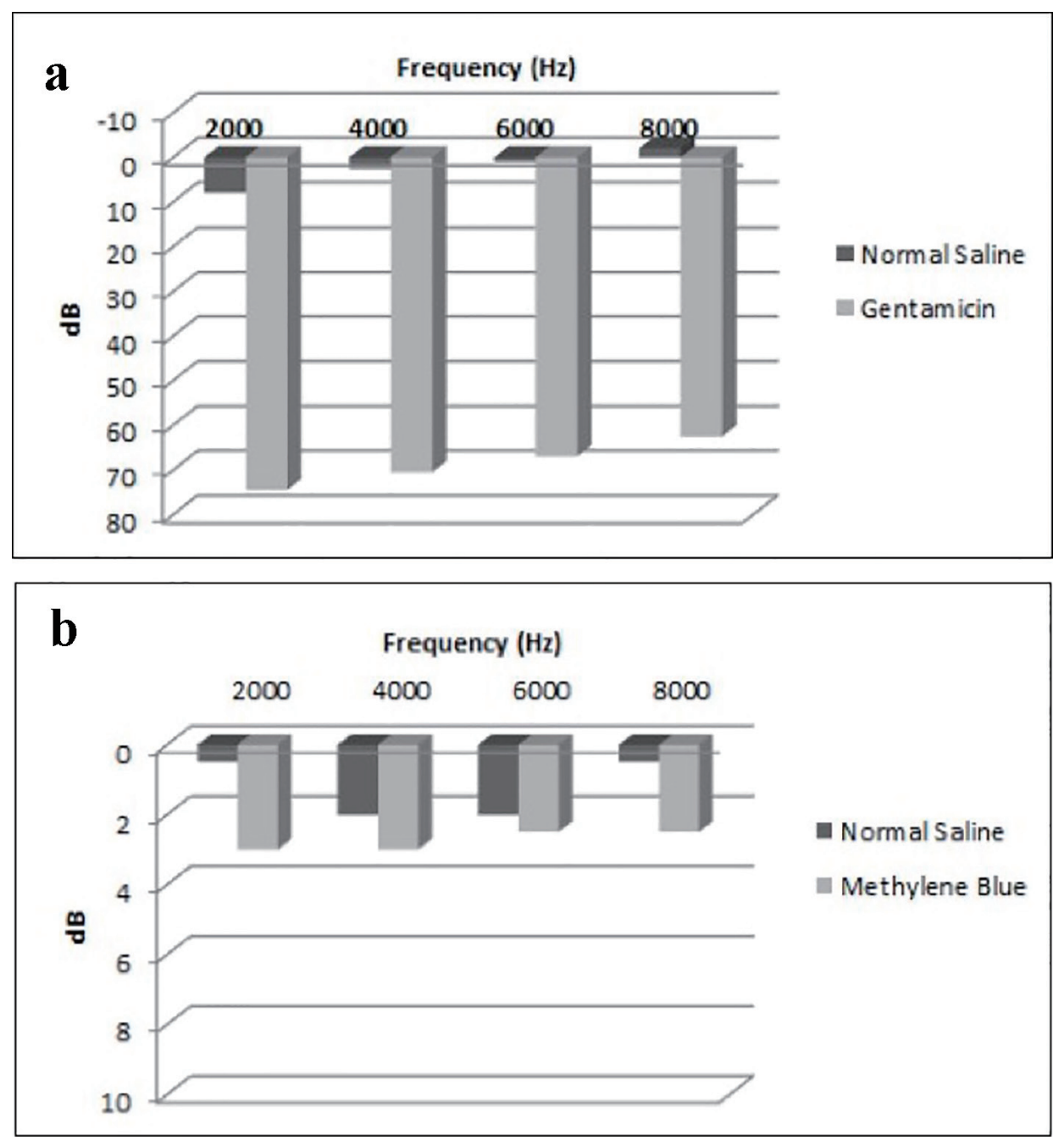

Figure 2. Mean threshold of differences in each group. (a) Mean threshold of differences in the control group (normal saline vs. gentamicin). (b) Mean threshold of differences in the experimental group (normal saline vs. methylene blue). 


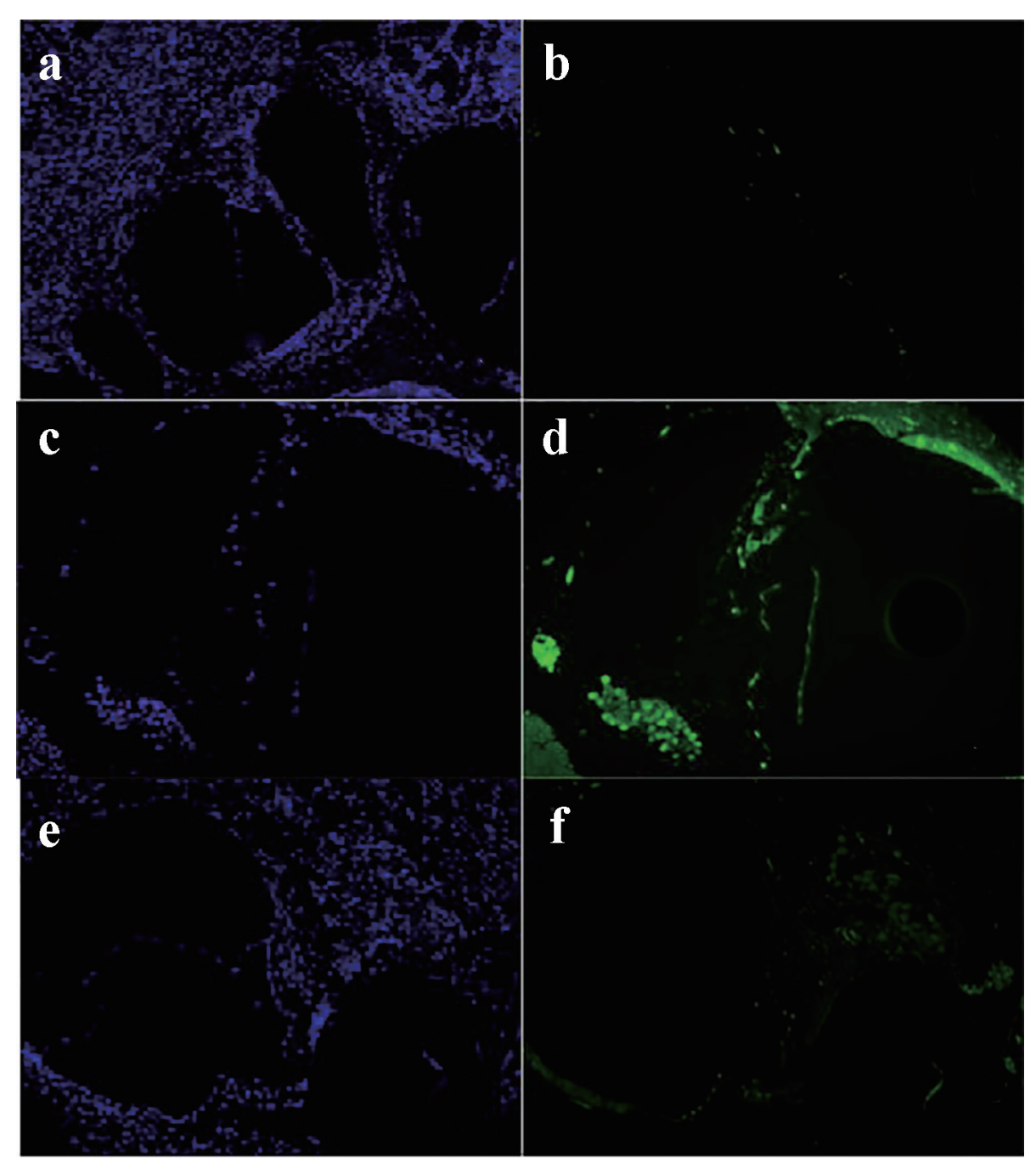

Figure 3. Normal saline group: DAPI stain (a) and FITC stain (b). Gentamicin group: DAPI stain (c) and FITC stain (d). Methylene blue group: DAPI stain (e) and FITC stain $(\mathrm{g})(\times 20)$. DAPI: 4',6-diamidino-2-phenylindole; FITC: fluorescein isothiocyanate.

\section{Statistical analysis}

We compared the mean threshold of hearing loss in each group. In the control group, the mean threshold of hearing loss difference for all the frequencies combined was $66.25 \mathrm{~dB}(\mathrm{P}$ $<0.001,95 \%$ CI: 44.6 - 87.9). In the experimental group, the mean threshold of hearing loss difference for all the frequencies combined was $1.50 \mathrm{~dB}$ and was not found to be statistically significant ( $\mathrm{P}=0.688,95 \% \mathrm{CI}$ : -6.8 to 9.8$)$.

All thresholds of hearing loss regarding each frequency in both the control and the experimental groups are shown in Figure 2.

\section{Histology and IHC}

Initial DAPI is a fluorescent stain that binds strongly to A and $\mathrm{T}$ regions in DNA. DAPI staining was achieved to assess the architectural structures of all specimens (blue light).
FITC staining is a derivative from fluorescein. FITC staining (green light) indicates uptake of caspase-3, and thus cell apoptosis. No significant cell apoptosis was detected in the normal saline group and methylene blue groups. However, the gentamicin group showed high uptake of caspase-3 antibody (Fig. 3).

\section{Discussion}

As mentioned earlier, methylene blue toxicity remains a rare condition. In fact, most adverse events reported are benign, such as pain at the site of injection or pseudo cyanosis, a blue discoloration of the skin and mucosa and a green coloration of the urine. However, when used in high doses, more severe events may be present. These may consist of cardiac arrhythmias, pulmonary edema or renal insufficiency. Furthermore, allergy to this agent is possible, and cases of anaphylactic or urticarial reactions were reported. Majithia and Stearns [19] 


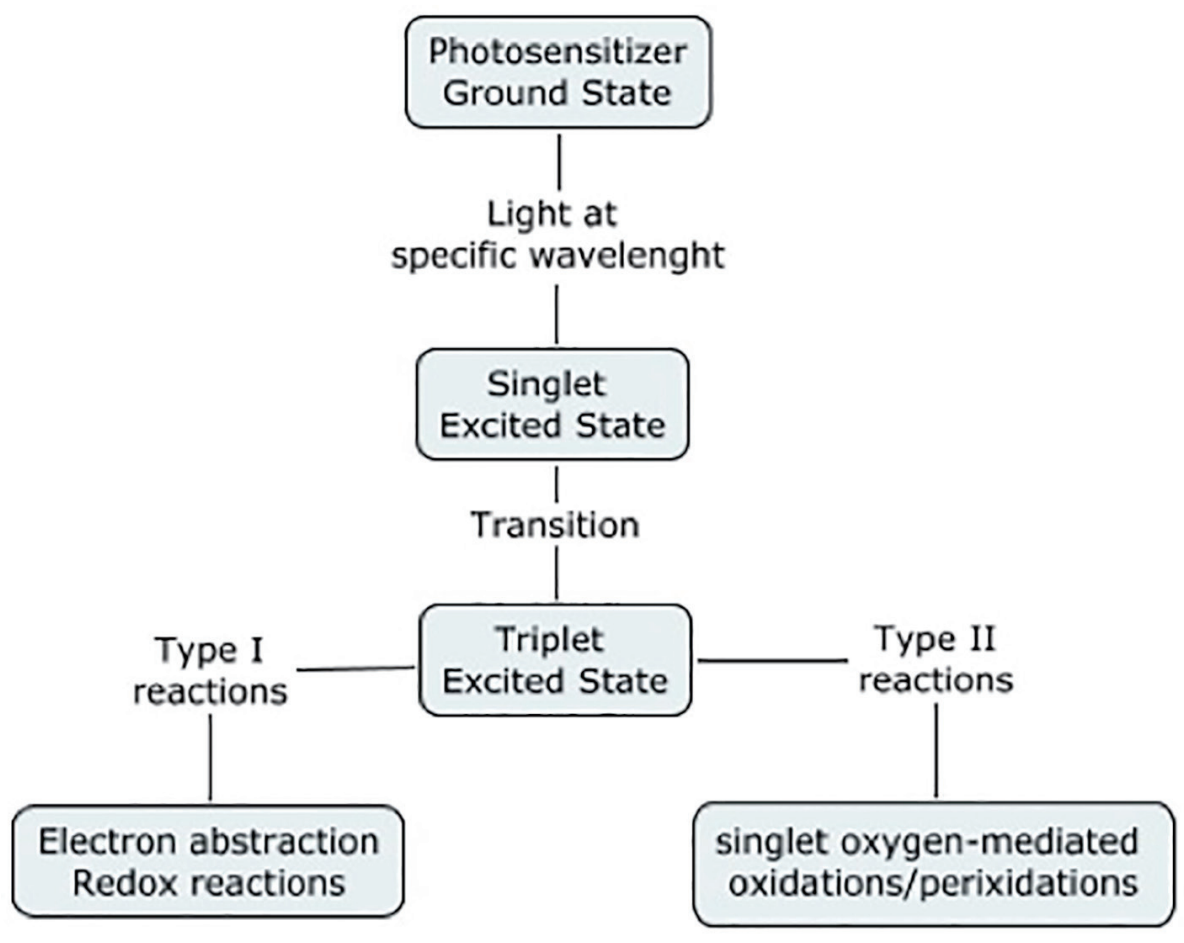

Figure 4. Photodynamic therapy. Adapted from Wainwright (1998).

reported a case of toxicity to methylene blue following its use to localize a parathyroid adenoma. Symptoms of confusion, aphasia and disinhibition following infusion were described, but the patient recovered within $48 \mathrm{~h}$.

In the otological field, methylene blue use was described in tympanoplasty. In fact, Wong (1983) [20] or Talas et al (2008) [21] reported its use to facilitate grafts placement. Vaiman et al [22] investigated the usefulness of the methylene blue staining for the operation of tympanoplasty in surgical training process. The authors used intraoperative staining of tympanoplasty grafts with methylene blue in one group, and no staining of the grafts in the other group. The pure-tone audiogram testing revealed significant improvement of hearing in all successful cases.

The emergence of resistant strains of bacteria is becoming increasingly challenging. In fact, drug resistance with a wide variety of pathogens, such an MRSA, poses a challenge to healthcare, and is associated with high morbidity. Many researches have been done in the past decade to find alternative ways to control infections. Photodynamic therapy uses a molecule, such as an aromatic molecule, which absorbs light of certain energy [16]. It then undergoes an electronic transition to the singlet excited state (electron spin paired). The molecule can either lose its energy and thus return to its ground state, or it may undergo a transition to the triplet-excited state (electron spins unpaired). Then again, it may return to its ground state, it may undergo redox reactions with the environment, or its energy may be transferred to molecular oxygen, leading to the formation of the labile singlet oxygen, resulting in bacteriolysis (Fig. 4).

Methylene blue is part of the phenothiazinium family (along with toluidine blue $\mathrm{O}$ ), which are blue dyes. It has been used widely in histology over the past century. In fact, the photodynamic action of methylene blue against bacteriophages and viruses was first described by Schultz and Krueger (1928) [23].

PDT has been shown to be effective against a wide range of pathogens in vitro. In fact, it has been shown to significantly eradicate Staphylococcus sp [14, 23, 24], Pseudomonas sp 6 , or Candida sp [25]. Many studies have also shown the efficacy of PDT using in vivo models. Zolfaghari et al [9] demonstrated that in vivo PDT was effective at reducing the total number of viable MRSA in mouse wound models. Furthermore, histological examination of the wounds showed neither collateral tissue necrosis nor architectural disturbances.

This study is the first to primarily assess the ototoxic potential of methylene blue. It was not found to be ototoxic in our study, as it was not associated with an increase in thresholds in all frequencies tested, and no significant apoptotic changes were found by immunohistochemistry.

\section{Conclusion}

Methylene blue is widely used in the medical field to treat various pathologies. PDT is a promising alternative in the control of local infections. No ototoxic effect was demonstrated in our study with the utilization of methylene blue not photoactivated. As PDT is associated with the production of free radicals and reactive oxygen species, further studies to determine the safety and utilization of methylene blue once photoactivated are needed. 


\section{Sources of Financial Support}

None.

\section{Funding}

No funding was received for this work from any of the following organizations: NIH, Welcome Trust, HHMI or other.

\section{Conflict of Interest}

None.

\section{References}

1. Caro, H. Improvement in the Production of Dye-Stuffs from Methyl-Aniline. Patent No. 204,796. Mannheim: U.S. Patent Office. 1878.

2. Cooper MS, Randall M, Rowell M, Charlton M, Greenway A, Barnes C. Congenital methemoglobinemia type II-clinical improvement with short-term methylene blue treatment. Pediatr Blood Cancer. 2016;63(3):558-560.

3. Tataru AP, Warrick BJ, Smolinske S. A systematic analysis of methylene blue for drug-induced shock in humans. Clin Toxicol (Phila). 2017;55(3):228.

4. Groneberg DA, Fischer A. Methylene blue improves the hepatopulmonary syndrome. Ann Intern Med. 2001;135(5):380-381.

5. Ehrhardt K, Davioud-Charvet E, Ke H, Vaidya AB, Lanzer M, Deponte M. The antimalarial activities of methylene blue and the 1,4-naphthoquinone 3-[4-(trifluoromethyl) benzyl]-menadione are not due to inhibition of the mitochondrial electron transport chain. Antimicrob Agents Chemother. 2013;57(5):2114-2120.

6. Salman R, Sebaaly MG, Wehbe MR, Sfeir P, Khalife M, Al-Kutoubi A. Preoperative Localization of Mediastinal Parathyroid Adenoma with Intra-arterial Methylene Blue. Cardiovasc Intervent Radiol. 2017;40(6):953-956.

7. Ginimuge PR, Jyothi SD. Methylene blue: revisited. J Anaesthesiol Clin Pharmacol. 2010;26(4):517-520.

8. Silva ZS, Jr., Huang YY, de Freitas LF, Franca CM, Botta SB, Ana PA, Mesquita-Ferrari RA, et al. Papain gel containing methylene blue for simultaneous caries removal and antimicrobial photoinactivation against Streptococcus mutans biofilms. Sci Rep. 2016;6:33270.

9. Zolfaghari PS, Packer S, Singer M, Nair SP, Bennett J, Street C, Wilson M. In vivo killing of Staphylococcus aureus using a light-activated antimicrobial agent. BMC Microbiol. 2009;9:27.

10. Ng R, Singh F, Papamanou DA, Song X, Patel C, Holewa
C, Patel N, et al. Endodontic photodynamic therapy ex vivo. J Endod. 2011;37(2):217-222.

11. Rai M, Spratt D, Gomez-Pereira PR, Patel J, Nair SP. Light activated antimicrobial agents can inactivate oral malodour causing bacteria. J Breath Res. 2016;10(4):046009.

12. Wilson M, Yianni C. Killing of methicillin-resistant Staphylococcus aureus by low-power laser light. J Med Microbiol. 1995;42(1):62-66.

13. Wainwright M, Phoenix DA, Laycock SL, Wareing DR, Wright PA. Photobactericidal activity of phenothiazinium dyes against methicillin-resistant strains of Staphylococcus aureus. FEMS Microbiol Lett. 1998;160(2):177181.

14. Nakonechny F, Firer MA, Nitzan Y, Nisnevitch M. Intracellular antimicrobial photodynamic therapy: a novel technique for efficient eradication of pathogenic bacteria. Photochem Photobiol. 2010;86(6):1350-1355.

15. Biel MA, Sievert C, Usacheva M, Teichert M, Balcom J. Antimicrobial photodynamic therapy treatment of chronic recurrent sinusitis biofilms. Int Forum Allergy Rhinol. 2011;1(5):329-334.

16. Peloi LS, Soares RR, Biondo CE, Souza VR, Hioka N, Kimura E. Photodynamic effect of light-emitting diode light on cell growth inhibition induced by methylene blue. J Biosci. 2008;33(2):231-237.

17. http://www.ventana.com/product/203?type=204.

18. Snedecor GW, Cochran WG. Statistical Methods. 8th Ed. Ames: Iowa State Press; 1989.

19. Majithia A, Stearns MP. Methylene blue toxicity following infusion to localize parathyroid adenoma. J Laryngol Otol. 2006;120(2):138-140.

20. Wong RT. Methylene blue stained temporalis fascia graft in tympanoplasty. Laryngoscope. 1983;93(2):241.

21. Talas D, Nguyen-Huynh A, Blevins NH. Intraoperative staining of tympanoplasty grafts: a technique to facilitate graft placement. Otolaryngol Head Neck Surg. 2008;138(5):682-683.

22. Vaiman M, Sarfaty S, Gavriel H, Kraus M, Kaplan D, Puterman M. Intravital staining with methylene blue in tympanoplasty. Eur Arch Otorhinolaryngol. 2010;267(9):1351-1354.

23. Schultz EW. Krueger AP. Inactivation of staphylococcus bacteriophage by methylene blue. Proceedings of the Society of Experimental Biology and Medicine. 1928;26:100-101.

24. Miyabe M, Junqueira JC, Costa AC, Jorge AO, Ribeiro MS, Feist IS. Effect of photodynamic therapy on clinical isolates of Staphylococcus spp. Braz Oral Res. 2011;25(3):230-234.

25. Souza RC, Junqueira JC, Rossoni RD, Pereira CA, Munin E, Jorge AO. Comparison of the photodynamic fungicidal efficacy of methylene blue, toluidine blue, malachite green and low-power laser irradiation alone against Candida albicans. Lasers Med Sci. 2010;25(3):385-389. 